\title{
CT-Scans of Cochlear Implant Patients with Characteristics of Pendred Syndrome
}

\author{
Sebastian Roesch Gerhard Moser Gerd Rasp Miklós Tóth
}

Department of Otorhinolaryngology, Head and Neck Surgery, General Hospital Salzburg, University Hospital of Paracelsus Medical University Salzburg, Salzburg, Austria

\section{Key Words}

Sensorineural hearing loss $•$ Enlarged vestibular aqueduct $\bullet$ Mondini malformation $\bullet$ CT-scans - Pendred syndrome • Cochlear implant

\begin{abstract}
Background: Sensorineural hearing loss (SNHL) in newborns is estimated with an incidence around 1:10,000 per year and is divided into syndromic and non-syndromic forms. In case of present retrocochlear function, cochlear implantation allows speech and cognitive development in affected children, comparable to that of normal hearing children. Pathogenesis of SNHL remains unclear in many cases. Imaging of the temporal bone, such as computed tomography (CT) and magnetic resonance imaging (MRI), can reveal conspicuous findings, e.g. enlarged vestibular aqueduct (EVA) and Mondini malformation (MM) of the cochlea. These malformations can be a clinical sign for Pendred syndrome. Methods: We screened CT scans of 75 cochlear implant patients for EVA and MM. Results: Six patients were observed to have either EVA alone $(n=3)$, or MM alone $(n=2)$, or a combination of both $(n=1)$. Further malformations of the temporal bone could be found within the whole group, as well. Conclusion: Our results confirm the general opinion on EVA and MM, being commonly found in patients with SNHL. A possible association with Pendred syndrome needs to be confirmed by genetic investigations with search for mutations in the SLC26A4 gene and further clinical tests, such as perchlorate test for surveillance of thyroid function.
\end{abstract}




\section{Cellular Physiology and Biochemistry}

Cell Physiol Biochem 2013;32(suppl 1):166-172

\begin{tabular}{l|l}
\hline DOI: $10.1159 / 000356636$ & (c) 2013 S. Karger AG, Basel
\end{tabular}

Publisned online: December 18, $2013 \quad$ www.karger.com/cpb

Roesch et al.: CT-Scans With Characteristics of Pendred Syndrome

\section{Introduction}

From a clinical point of view, the diagnosis of congenital or progressive sensorineural hearing loss (SNHL) within newborn screening, leads to the question of therapeutic opportunities at first sight. Fortunately, there are versatile technical devices such as hearing aids and cochlear implants, affording speech and cognitive development, comparable to that of normal-hearing individuals. With the help of genetic testing and an ongoing identification of specific mutations, the question on the etiological background of SNHL comes to the fore.

The clinical symptom of severe SNHL or deafness in children has a genetic background in about $65 \%$ of the cases, with $85 \%$ of them in a non-syndromic appearance [1]. In case of specific accompanying symptoms, the hearing loss is defined as a syndromic form. The most common syndromic form of congenital SNHL or deafness is Pendred syndrome [2]. It is defined through congenital SNHL or deafness and a malfunction of thyroid hormone synthesis. The genetic background is a mutation of the SLC26A4 gene, coding for a transmembrane protein with anion transport function, called Pendrin [3-5]. Pendrin is present in multiple organs, such as the kidney, the airways, the thyroid gland and the inner ear. Besides maintenance of ion composition and $\mathrm{pH}$ homeostasis between separated compartments in different organs [5], Pendrin plays a special role during embryological development of the inner ear [3]. The knowledge on the function of Pendrin within the inner ear and inner ear development is based mainly on mouse models [6].

The over 160 known mutations of the SLC26A4 gene [4] can appear in a monoallelic or a biallelic fashion and lead to non-syndromic enlarged vestibular aqueduct (EVA) or Pendred syndrome [5]. Pendred syndrome is typically associated with biallelic mutations, whereas monoallelic mutations may lead to SNHL without further phenotypes [5]. However, a mutation of the SLC26A4 gene is not mandatory in case of present SNHL. The distribution of SLC26A4 mutations among patients with SNHL differs among different ethnic populations worldwide. In Asian populations mutations are found with a much higher incidence compared to Caucasian patients [7].

In the context of clinical evaluation of SNHL in general, imaging modalities, such as CT scan and MRI of the temporal bone, play a significant role. Possible morphological variations and malformations can be visualized and may lead to distinct diagnosis or give at least a hint for possible diagnosis [8]. There are two radiological findings, which are known to be associated with mutations of the SLC26A4 gene, either in a monoallelic or a biallelic manner. First one is an EVA, which is the most common finding in temporal bone imaging [4, 9] in case of SNHL. EVA can be found either on one or both sides, however in case of Pendred syndrome, usually both sides are affected [9]. The definition of EVA is based on the description of Valvassori and Clemis [10], with a diameter of more than $1.5 \mathrm{~mm}$ at the midpoint. Normal vestibular aqueduct can hardly be identified completely on the CT scan or MRI and runs from the posterior surface of the temporal bone (operculum) towards the common crus of the superior and the posterior semicircular canal. It harbors the membranous endolymphatic duct. EVA could be found in numerous CT scan investigations of children with SNHL, not necessarily being diagnosed as Pendred syndrome according to genetic testing. In these cases EVA is defined as non-syndromic EVA [4].

A second possible sign for Pendred syndrome, revealed by imaging, is Mondini malformation (MM). A conclusive definition for MM does not exist. It is more like a descriptive term for a crude appearance of the cochlea, without clearly definable 2.5 turns, a missing modiolus and possible accompanying enlargement of the vestibule.

Both malformations, EVA and MM, are seen as diagnostic hints or radiologic markers for possible Pendred syndrome [10]. Concerning the pathogenesis of hearing loss in patients with proven EVA, EVA seems not to be the causative factor for the hearing loss, but more an accompanying morphological result of an altered fluid balance in the inner ear during embryological development, due to malfunction of Pendrin [10]. 


\section{Cellular Physiology

Cell Physiol Biochem 2013;32(suppl 1):166-172

Roesch et al.: CT-Scans With Characteristics of Pendred Syndrome

\section{Materials and Methods}

We reviewed temporal bone CT scans of 75 patients having severe SNHL. Gender distribution was $46.6 \%$ male patients $(n=35)$ and $53.3 \%$ female patients $(n=40)$ with age ranging from 13 months up to 84 years. All patients received cochlear implantation, either on one side or both, at our clinics between years 2009 and 2011.The CT scans were performed routinely prior to surgery, in order to get preoperative anatomical information. Imaging was performed with Phillips Brilliance ${ }^{\circledR}$ 64-channel CT scanner with section- thickness of $1 \mathrm{~mm}$ and image reconstruction in axial and coronal planes. In some cases postoperative control CT scans existed as well, which were reviewed, too. First, generally conspicuous findings were separated from physiological findings and reviewed again in detail in a second step. Conspicuous radiological findings of either one or both sides were specifically screened for EVA and MM within the second step. Findings, other than EVA or MM, were described anatomically and separated into subgroups, depending on the affected area within the temporal bone. In case of EVA and MM, we checked for altered laboratory parameters concerning the thyroid gland, such as thyroid stimulating hormone (TSH), free thyroxine (fT4) and free triiodothyroine (fT3). Perchlorate testing is not an investigation routinely performed at our clinics, and was not performed in any of the cases.

Informed consent for anonymous retrospective data analysis was not obtained, correspondent to guidelines of the local ethics commission.

\section{Results}

We investigated preoperative, and if existing, postoperative CT scans of 75 patients, who received cochlear implantation, 53.3\% were female, 46.6\% male, age ranging between 13 months and 84 years. After a first view we defined 27 cases (36.0\%) of conspicuous findings, affecting any structure of the temporal bone. After first review, we excluded two patients, both of them showing radiological characteristics of cochlear otosclerosis, and defined different subgroups for the remaining patients, depending on the morphological structure affected.

In fourteen cases, we could find a malformation of any structure of the inner ear and the internal auditory canal. In most of the cases the internal auditory canal revealed normal width in the axial and coronal planes. Three temporal bones had an altered width on both sides with two of them enlarged with more than $5 \mathrm{~mm}$ and one very narrow, with a diameter of $2 \mathrm{~mm}$. In case of normal temporal bone, there is no direct connection between the inner ear and the internal auditory canal. In two patients we could find such a connection, one small and one large bony dehiscence between the vestibule and the internal auditory canal on both sides. Both connections were found at the fundus of the internal auditory canal, where the superior vestibular nerve enters the vestibule (Fig. 1A,B). The facial canal physiologically begins at the fundus of the internal auditory canal, forming the so-called meatal foramen. The labyrinthine portion of the facial nerve lies directly on the cochlea and has a curved run. In our cohort of patients, we could find enlarged and very narrow facial canals between the vestibule and the cochlea (Fig. 1C,D). In one case, a narrow, curved bony canal could be found between the fundus of the internal auditory canal and the tympanic portion of the facial canal. The proximal end of this canal lies immediately posterior to the labyrinthine portion of the facial canal. In this case the bony channel for the superior vestibular nerve was not clearly visible (Fig. 1E). One patient had moderate vertigo after cochlear implantation. The postoperative CT scan could reveal a protrusion of the cochlear implant electrode into the internal auditory canal through a presumably preexisting bony dehiscence between the end portion of the first turn of the cochlea and the internal auditory canal (Fig. 1F).

Three patients showed alterations of the whole bony structure of the temporal bone with poor pneumatization and a thick and compact otic capsule, similar to the findings of a systemic disease like osteopetrosis or Paget disease. The CT scans were approximately similar on both sides in all of those three cases. 


\section{Cellular Physiology and Biochemistry}

Cell Physiol Biochem 2013;32(suppl 1):166-172

\begin{tabular}{l|l}
\hline DOI: $10.1159 / 000356636$ & (c) 2013 S. Karger AG, Basel
\end{tabular}

Roesch et al.: CT-Scans With Characteristics of Pendred Syndrome

Fig. 1. Horizontal CT scans of six different cases, showing variable temporal bone malformations. (A, B) Two right temporal bones, with a bony dehiscence (white horizontal arrows) between the vestibule and the internal auditory canal. (C, D) The labyrinthine portion of the facial canal at the meatal foramen (white oblique arrows) of two different patients, representing different diameters from very wide (C, right temporal bone) to very narrow (D, left temporal bone). (E) An additional bony canal (BC) can be seen in a left temporal bone between the internal auditory canal and the tympanic portion of the facial canal. (F) The postoperative CT scan of a right temporal bone reveals a bony dehiscence (black vertical arrow) between the internal auditory canal and the cochlea, with protrusion of the cochlear electrode.

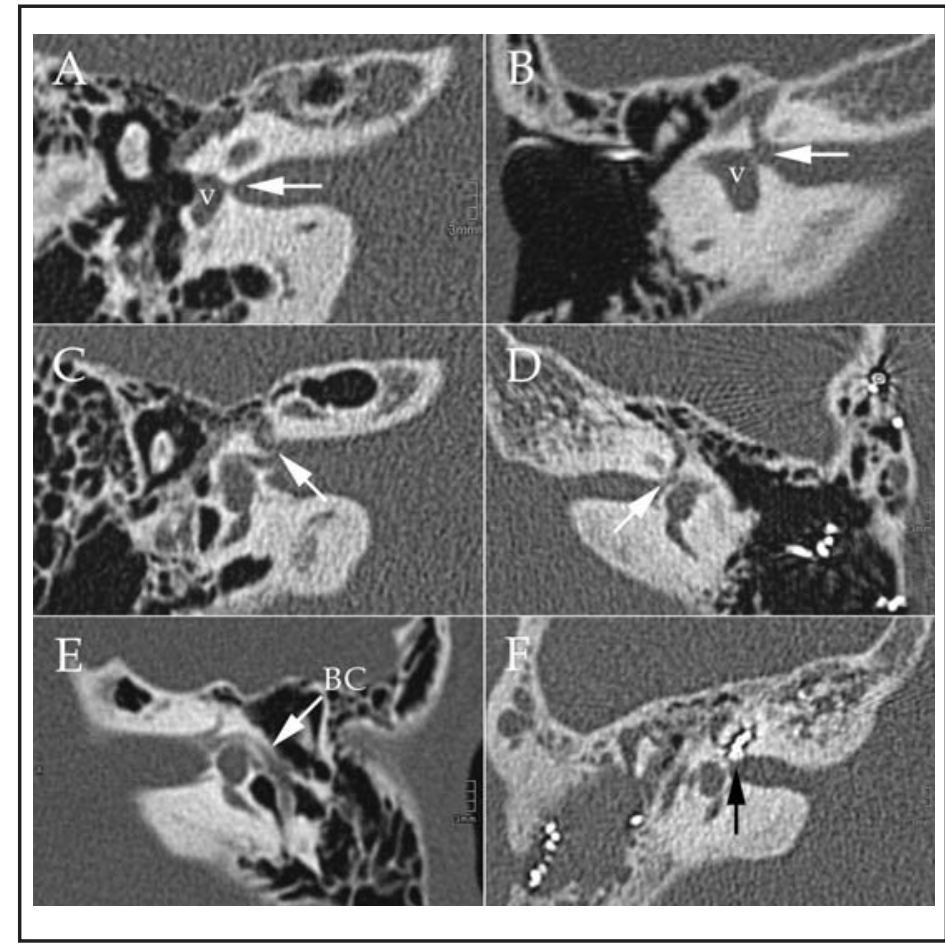

Table 1. Patients with radiological findings with characteristics of Pendred syndrome: demographic data - age/gender; type of malformation - Mondini malformation (MM), Enlargement of vestibular aqueduct (EVA); sides affected

\begin{tabular}{cccc}
\hline Patient number & Age, years/gender & Type of malformation & Sides affected \\
\hline 1 & $6 /$ male & MM & both \\
2 & $7 /$ male & MM & both \\
3 & $17 /$ male & EVA & both \\
4 & $27 /$ female & EVA & both \\
5 & $36 /$ female & EVA & both \\
6 & $4 /$ female & MM + EVA & both \\
\hline
\end{tabular}

Another three patients had malformations of the middle ear, affecting the ossicles $(\mathrm{n}=2)$ or the structure of the tympanic cavity $(n=1)$. Concerning the affected ossicles malleus and incus showed unusual appearance on both sides in both of the cases.

Concerning EVA and MM, we found six patients within the whole cohort of 75 patients, representing 8.0\% (Tab. 1). Three patients showed EVA only, on both sides. Two of them additionally had a high jugular bulb on the right side with a bony dehiscence between the jugular bulb and the endolymphatic duct (Fig. 2A,B). Three patients had a malformation of the inner ear on both sides, fitting to the definition of MM. Two patients showed MM only and one patient had both, EVA and MM on both sides (Fig. 3). In all six cases, both sides were affected. Laboratory results for TSH, fT4 and fT3 of all six patients were all within normal range. Clinically, there was no sign for goiter in any of the patients, as well. Epidemiological data of these six patients can be found in Table 1. Five of the patients had congenital moderate to severe sensorineural hearing loss, at least on one side; only one patient showed progressive hearing loss during childhood (Tab. 2).

During cochlear implant operation, we found a Gusher phenomenon in all three patients with MM. Gusher phenomenon is defined as an active efflux of perilymph after cochleostomy due to reasonable advanced communication between the inner ear and the cerebral space. 


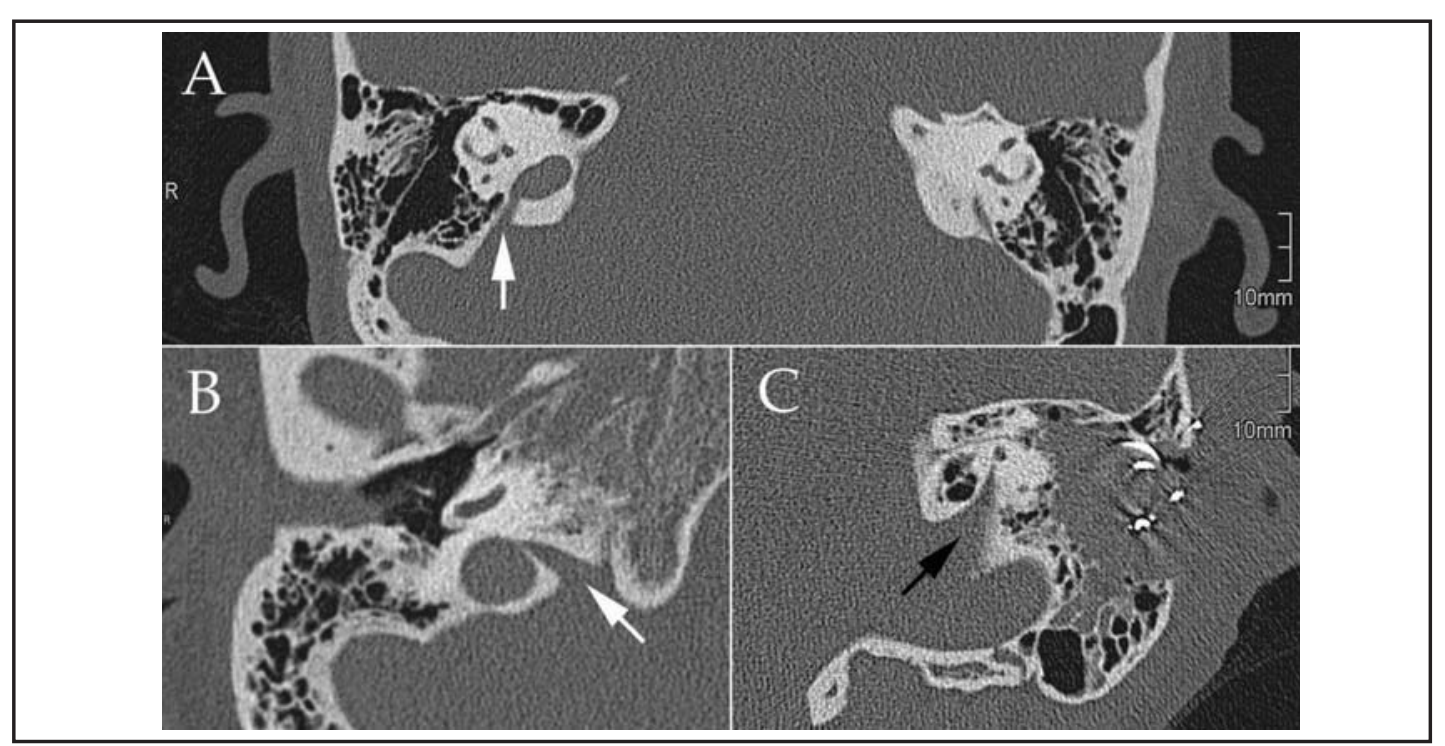

Fig. 2. Different forms of enlargement of vestibular aqueduct (EVA) are presented in three different patients with EVA only. (A) Axial computed tomography scan of both temporal bones with EVA on both sides (vertical arrow indicating EVA of the right ear). The endolymphatic duct has a direct connection with the high jugular bulb through a bony dehiscence. (B) Axial CT scan of a right temporal bone with EVA, also demonstrating a small bony dehiscence (white oblique arrow) allowing direct connection between the endolymphatic duct and the high jugular bulb. (C) Further aspects of an EVA (black oblique arrow) can be seen in the coronal CT scan of a left temporal bone, in this case without any bony arrosion of the posterior semicircular canal.

Fig. 3. Three axial CT scans, demonstrating different forms of the Mondini malformation. (A) There is no sign of the modiolus or spiral bony lamina; the cochlea (black oblique arrows) seems to be a common cavity on both sides. Only the basal turn can be identified, the other turns form a ball shaped cavity. (B) Lateral semicircular canal of the right side (white vertical arrow) with a mild malformation. The perilymphatic space has a large ampullary part and smaller extensions in the simplex part. The cochlea demonstrates a common cavity (not seen in this picture). (C) Left temporal bone, the cochlea also has no modiolus or spiral bony lamina. The vestibule and the semicircular canals show no malformation.

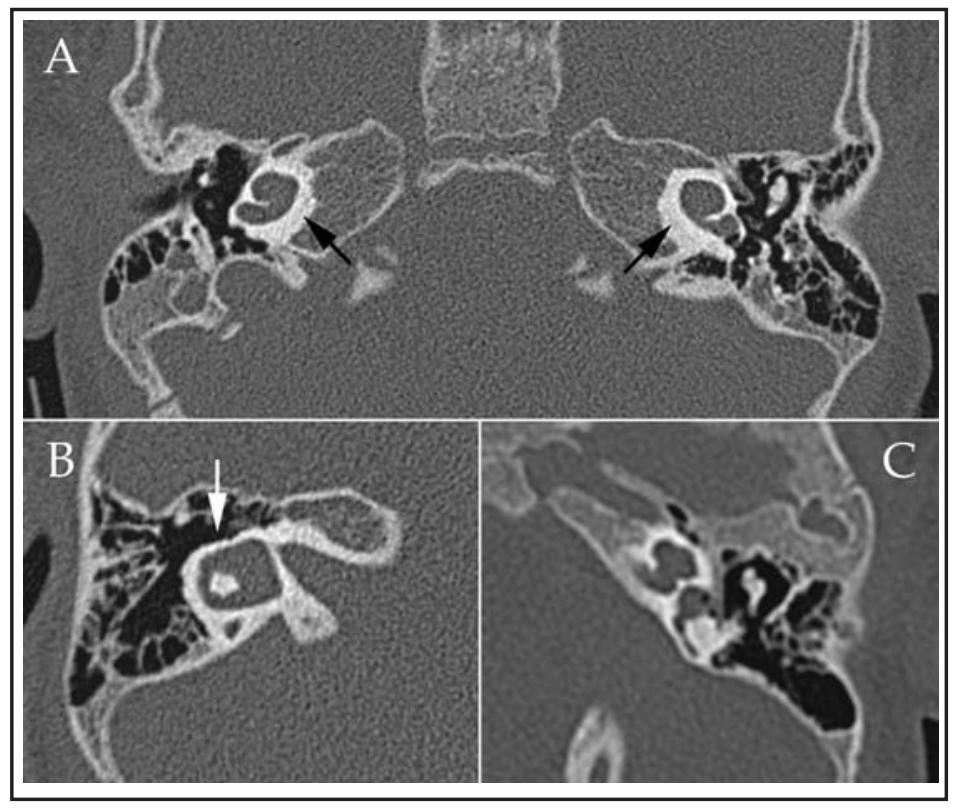

\section{Discussion}

The variety of anatomical malformations of the temporal bone in children with sensorineural hearing loss is large. Our findings from 75 patients confirm this in a distinct way. In spite of these findings, the causative reason for the malformation and the hearing loss remains unclear in most of the cases. Morphological malformations, found in radiological investigations can be a possible hint for altered development, leading to clinical symptoms. In case of Pendred syndrome, the typical radiological findings are EVA and MM of both sides. 


\section{Cellular Physiology and Biochemistry}

Cell Physiol Biochem 2013;32(suppl 1):166-172

\begin{tabular}{l|l}
\hline DOI: $10.1159 / 000356636$ & (C) 2013 S. Karger AG, Basel
\end{tabular}

Publisned online: December 18, $2013 \quad$ www.karger.com/cpb

Roesch et al.: CT-Scans With Characteristics of Pendred Syndrome

\begin{tabular}{ccc}
\hline Patient number & SNHL - sides/course/extent & Thyroid dysfunction \\
\hline 1 & both/congenital/severe & none \\
2 & both/congenital/severe & none \\
3 & right/congenital/severe; left/congenital/moderate & none \\
4 & right/congenital/deafness; left/congenital/moderate & none \\
5 & both/progressive/severe & none \\
6 & both/congenital/severe & none \\
\hline
\end{tabular}

Table 2. Evaluation of sensorineural hearing loss (SNHL) and thyroid dysfunction within the group of patients with EVA and/or MM: SNHL - sides (left, right, both)/course (congenital or progressive)/ extent (mild, moderate, severe, deafness); Thyroid dysfunction - altered laboratory values for TSH, fT3 and fT4

We could find six patients with one or more of those malformations on both sides. In order to support the diagnosis of possible Pendred syndrome, we evaluated laboratory results for TSH, fT4 and fT3. Laboratory results of thyroid gland function parameters were all within normal range in all six patients having EVA and/or MM (Table 2). There was no laboratory sign for hypothyroidism, a thyroid malfunction which is known to be found in some patients with Pendred syndrome, due to impaired iodine organification within thyrocytes due to malfunction of Pendrin [11]. We were not able to perform a perchlorate discharge test, due to the absence of an established testing at our clinics. The test would have given proof for presence or absence of a malfunction of iodine organification in our patients. Even in patients with proven biallelic mutations of the SLC26A4 gene and a partial organification defect for iodine, goiter or hypothyroidism is developed only rarely [11]. Therefore, we cannot prove a malfunction of iodine organification in our group of patients, but normal laboratory parameters for TSH, fT4 and fT3 do not exclude the possibility of Pendred syndrome.

Additionally to EVA and MM, our CT-scan collection revealed multiple unusual inner ear malformations, e.g. a bony dehiscence between the vestibule and the internal auditory canal and between the cochlea and the internal auditory canal. All of those inner ear malformations, including EVA and MM, might be a result of a defect within the development of the bony otic capsule. Generally, the fully developed otic capsule is a thick bony plate, with two natural exceptions at the upper part of the fundus of the internal auditory canal. Anteriorly there is a bony thinning between the cochlea and the labyrinthine portion of the facial nerve, and posteriorly, there is a thinning of the bone between the vestibule and the superior vestibular nerve. In case of Pendred syndrome, only a thin bony otic capsule develops around the perilymphatic space and the modiolus is commonly absent, due to processes during development, not completely understood, yet. From a clinical point of view, it seems reasonable that the dehiscences found in our patients at the regions of generally thin otic capsule might be a result of the generally reduced ossification of the otic capsule.

Concerning the detection and evaluation of hypoplastic bony structures such as dehiscences or an absent modiolus in a CT scan, it is important to note that imaging of structures with a size smaller than about $0.2 \mathrm{~mm}$ is limited due to resolution of the imaging device. However, in our case, we found hypoplastic malformations of the modiolus and bony dehiscences of the bony capsule. The fact that these structures are hypoplastic or of altered appearance needs to be evaluated as pathologic, independently from the fact whether they are really absent, just hypoplastic or not displayed by the imaging device.

Overall, based on our CT-scan results, we assume that a bony dehiscence, between the internal auditory canal and the vestibule, as well as between the internal auditory canal and the cochlea, might be an additional morphological sign for possible malfunction of Pendrin during development of the temporal bone. Minor variations within the otic capsule may be easily overseen and therefore have not been mentioned, yet in the context of Pendred syndrome. There are examples for a retrospective detection of a radiological correlate for a clinical symptom like the dehiscence of the anterior semicircular canal, which was found as a radiological sign for dizziness just 15 years ago by Minor et al. [12]. 


\section{Cellular Physiology and Biochemistry}

Cell Physiol Biochem 2013;32(suppl 1):166-172

DOI: $10.1159 / 000356636$

(c) 2013 S. Karger AG, Basel

www.karger.com/cpb

Roesch et al.: CT-Scans With Characteristics of Pendred Syndrome

In order to give proof of a possible association between our findings described and possible mutations in SLC26A4 gene or Pendred syndrome, we will have to perform genetic testing in a next step, after approval of the local ethics commission.

\section{Conclusion}

The aim of our study was to obtain an overview on the presence of radiological characteristics for Pendred syndrome among patients with severe sensorineural hearing loss, who received cochlear implantation between the years 2009 and 2011 at our clinics. Definite diagnosis of Pendred syndrome lacks genetic testing and evaluation of iodide organification in our study. However, we were able to identify six patients showing radiological characteristic of Pendred syndrome who will now be administered to specific genetic testing for mutations of SLC26A4 gene. We could find no further characteristic for Pendred syndrome in any of our patients, such as goiter or hypothyroidism. This does not exclude the possibility for Pendred syndrome in our patients due to the fact that a malfunction of iodine organification caused by impaired Pendrin function in the thyroid does not necessarily lead to manifest hypothyroidism or goiter.

Moreover, we were able to identify malformations of the temporal bone, not mentioned in the context of Pendred syndrome, yet. Bony dehiscences between the internal auditory canal and the vestibule or the cochlea might be another sign of impaired inner ear development and it seems reasonable, that similar pathological processes, like in the case of EVA or Mondini malformation, lead to an altered bony structure at further regions of the temporal bone.

\section{Conflict of Interests}

None.

\section{References}

1 Strutz J, Arndt O: Praxis der HNO-Heilkunde, Kopf- und Halschirurgie: 265 Tabellen. Stuttgart; New York NY, Thieme, 2010.

-2 Broomfield SJ, Bruce IA, Henderson L, Ramsden RT, Green KMJ: Cochlear implantation in children with syndromic deafness. Int J Pediatr Otorhinolaryngol 2013;77:1312-1316.

3 Wangemann P: The role of pendrin in the development of the murine inner ear. Cell Physiol Biochem 2011;28:527-534.

4 Ito T, Choi BY, King KA, Zalewski CK, Muskett J, Chattaraj P, Shawker T, Reynolds JC, Butman JA, Brewer CC, Wangemann P, Alper SL, Griffith AJ: SLC26A4 genotypes and phenotypes associated with enlargement of the vestibular aqueduct. Cell Physiol Biochem 2011;28:545-552.

5 Dossena S, Nofziger C, Tamma G, Bernardinelli E, Vanoni S, Nowak C, Grabmayer E, Kössler S, Stephan S, Patsch W, Paulmichl M: Molecular and functional characterization of human pendrin and its allelic variants. Cell Physiol Biochem 2011;28:451-466.

6 Kim H-M, Wangemann P: Failure of fluid absorption in the endolymphatic sac initiates cochlear enlargement that leads to deafness in mice lacking pendrin expression. PLoS ONE 2010;5:e14041.

7 Huang S, Han D, Yuan Y, Wang G, Kang D, Zhang X, Yan X, Meng X, Dong M, Dai P: Extremely discrepant mutation spectrum of SLC26A4 between Chinese patients with isolated Mondini deformity and enlarged vestibular aqueduct. J Transl Med 2011;9:167.

8 Joshi VM, Navlekar SK, Kishore GR, Reddy KJ, Kumar ECV: CT and MR imaging of the inner ear and brain in children with congenital sensorineural hearing loss. Radiographics 2012;32:683-698.

-9 Greinwald J, DeAlarcon A, Cohen A, Uwiera T, Zhang K, Benton C, Halstead M, Meinzen-Derr J: Significance of unilateral enlarged vestibular aqueduct. Laryngoscope 2013;123:1537-1546.

10 Griffith AJ, Wangemann P: Hearing loss associated with enlargement of the vestibular aqueduct: mechanistic insights from clinical phenotypes, genotypes, and mouse models. Hear Res 2011;281:11-17.

11 Bizhanova A, Kopp P: Controversies concerning the role of pendrin as an apical iodide transporter in thyroid follicular cells. Cell Physiol Biochem 2011;28:485-490.

12 Minor LB, Solomon D, Zinreich JS, Zee DS: Sound- and/or pressure-induced vertigo due to bone dehiscence of the superior semicircular canal. Arch Otolaryngol Head Neck Surg 1998;124:249-258. 